\title{
Julia Sets and Yang-Lee Zeros of the Potts Model on Bethe Lattices
}

\author{
Ruben Ghulghazaryan ${ }^{1}$, Nerses Ananikyan ${ }^{1,2}$, and Tore M. Jonassen ${ }^{3}$ \\ 1 Department of Theoretical Physics, Yerevan Physics Institute, \\ Alikhanian Brothers 2, 375036 Yerevan, Armenia \\ ghulr@moon. yerphi.am \\ 2 Institute of Physics, Academia Sinica, Taipei 11529, Taiwan \\ ananik@moon . yerphi .am \\ 3 Department of Computer Science, Oslo University College, \\ Cort Adelers gt. 30, N-0254 Oslo, Norway \\ torejo@hio.no
}

\begin{abstract}
Box counting fractal dimensions of Julia sets of the Q-state ferromagnetic Potts model on Bethe lattices are studied. It is shown that Julia sets are circles centered at the origin for values of magnetic field corresponding to the Yang-Lee zeros of the partition function. Also, for values of magnetic field from the Mandelbrot-like set on the complex magnetic field plane Julia sets are simply connected closed Jordan curves and for values of magnetic field outside the Mandelbrot-like set the Julia set is a Cantor set. Moreover, it is shown that the fractal dimension of a Julia set for a value of magnetic field corresponding to a Yang-Lee zero of the partition function is a local minimum as a function of magnetic field.
\end{abstract}

\section{Introduction}

The $Q$-state Potts model is a generalization of the Ising model to more-than-two components and has been a subject of an increasingly research interest over the last decades. An extended summary of results and a bibliography can be found in the review article by $\mathrm{Wu}[1]$ and references therein. The model has been studied on Bethe lattices also [2-8]. Bethe lattice is a topological abstraction compared to standard lattices. It corresponds to the interior of the infinitely extended Cayley tree with the coordination number $\gamma$ (Fig. 1). The surface of the Cayley tree cannot be neglected and leads to unusual behavior of models defined on a Cayley tree $[9,10]$. However, under certain conditions, exact results on Bethe lattices correspond to approximate results on standard lattices [11].

The Hamiltonian of the Potts model has the form

$$
-\beta \mathcal{H}=J \sum_{<i, j>} \delta\left(\sigma_{i}, \sigma_{j}\right)+h \sum_{i} \delta\left(\sigma_{i}, 1\right),
$$

where $\sigma_{i}$ takes the values $1,2,3, \ldots, Q$ and $\beta=1 / k T$. The first sum in the r.h.s. of (1) goes over all the edges and the second one over all the sites on the 


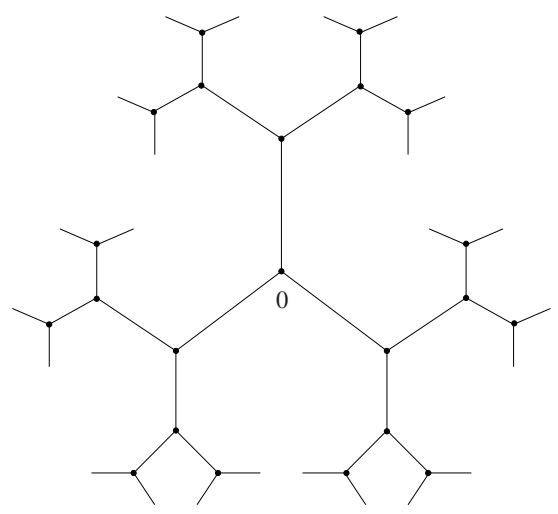

Fig. 1. A Cayley tree with coordination number $\gamma=3$.

lattice. Using the recursive structure of the Bethe lattice a recurrence relation for the partition function may be derived and the dynamical systems theory may be used for studying thermodynamic properties of the models. The recurrence relation has the form

$$
x_{n}=R\left(x_{n-1}\right), \quad R(x)=\frac{\mu+(z+Q-2) x^{\gamma-1}}{z \mu+(Q-1) x^{\gamma-1}},
$$

where $\mu=e^{h}$, and $z=e^{J}$. Here $\mathrm{x}$ is an auxiliary quantity that has no physical meaning but it determines the state of the system in the thermodynamic limit and thermodynamic quantities such as the magnetization, the specific heat and others may be expressed in terms of $\mathrm{x}[6,12,13]$. Later we will refer to (2) as the Potts-Bethe mapping.

The dynamics of the Bethe-Potts mapping has been studied in [6] and the Yang-Lee [14] zeros of the ferromagnetic Potts model was found. It has been shown that for $Q>1$ the Yang-Lee zeros are located on arcs of circles with the radius $R^{2}=\frac{(z+Q-2)^{\gamma}}{z^{\gamma}(Q-1)^{\gamma-2}}$. Moreover, in [6], for the first time, complex magnetic field metastability regions was introduced. According to the definition inside the metastability region there are two attracting fixed points and other fixed points are repelling. At the border of a metastability region one of the two attracting fixed points becomes a neutral (indifferent) fixed point [6]. Moreover, in [6] it was shown that the metastability region on the complex magnetic field plane is a part of a more complicated set - the Mandelbrot-like set of recursive mapping. For the first time Mandelbrot-like sets have been studied for multisite interaction Ising models on Cayley-type lattices $[12,13]$. By the definition the Mandelbrotlike set of a rational mapping $R$ is the set of parameter ( $\mu$ or $z$ ) values at which the mapping $R$ has neutral periodical points. From this definition it follows that the metastability region on the complex $\mu$-plane belongs to the inner part of the Mandelbrot-like set of recursive mapping. 
(a)

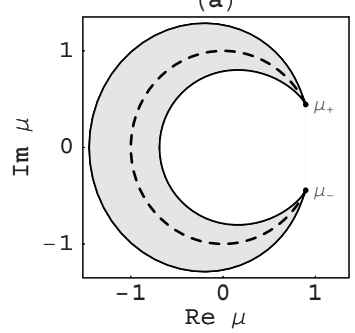

(b)

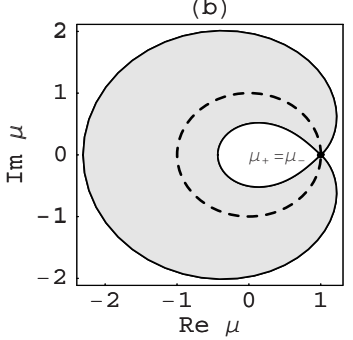

(c)

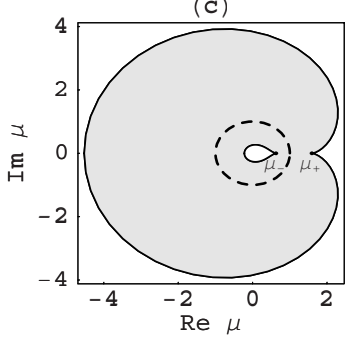

Fig. 2. Metastability regions and Yang-Lee zeros of the $Q$-state Potts model on the Bethe lattice with coordination number $\gamma=3$ and $Q=2$. The solid lines correspond to the boundary of metastability regions (gray filled areas). Dashed lines present an arc or circles of radius $R_{\mu}^{2}=\frac{(z+Q-2)^{\gamma}}{z \gamma(Q-1)^{\gamma-2}}$ and correspond to Yang-Lee zeros. (a) $T>T_{c}$ $(z=1.8), \mu_{ \pm}$are Yang-Lee edge singularity points; (b) $T=T_{c}$ critical point $\left(z=z_{c}=\right.$ 3); (c) $T<T_{c}(z=6), \mu_{ \pm}$are spinodal points of the model (for more details see [6]).

In 1994 Monroe proposed a criterion for finding the location of first order phase transition temperature for models on recursive lattices. According to this criterion the first order phase transition temperature defined from the condition that the absolute values of the derivatives of the recursive function in two attracting fixed points are equal [15]. Later Biskup et al. showed that the position of the partition function zeros is related to phase coexistence lines in the complex planes. The Yang-Lee zeros may be interpreted as first order phase transition points on the complex magnetic field plane. Hence, according to the theory of Biskup et al. [16] and Monroe's criterion [15] the Yang-Lee zeros lie on the line in the complex magnetic field plane where the derivatives of the recursive function in two attracting fixed points are equal. Thus, the Yang-Lee zeros are always located inside the metastability region (Mandelbrot-like set). Examples of metastability regions for different temperatures are given in Fig. 2 and an example of a Mandelbrot-like set of the Bethe-Potts mapping for $z=11$ and $Q=9$ is given in Fig. 3.a.

In spite of the dynamics of the Potts-Bethe mapping is studied for $Q>1$ case [6] very little is known about the Julia set of this mapping except for the $Q=2$ case corresponding to the Ising model [17]. A first attempt to fill this gap has recently been done by Monroe [18]. In his paper [18] Monroe showed numerically that the box counting fractal dimension of the Julia set of governing recurrence relation of a model on a recursive lattice is a minimum at a first order order phase transition $(\mu=1)$ as a function of temperature.

In this paper we investigate Julia sets of the Potts-Bethe mapping for complex values of $\mu$. In Section 2 we give an analytical treatment of properties of Julia sets of the Potts-Bethe mapping and describe some numerical algorithms for calculating fractal dimensions of Julia sets. In Section 3 results of numerical calculations are given. In the last Section a $\mathrm{C}$ code of program for calculating the fractal dimension of a Julia set is given. 

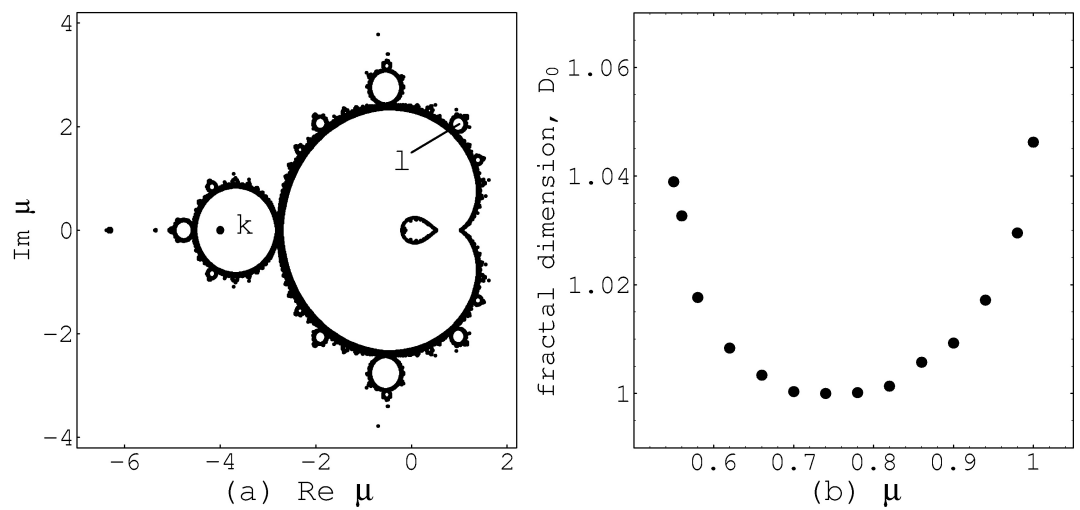

Fig. 3. (a) The boundary of Mandelbrot-like set of the Potts-Bethe mapping for $z=11$, $Q=9$ and $\gamma=3$. At the points $k(\mu=-4)$ and $l(\mu=1.0+2.0 I)$ outside of the metastability region the Potts-Bethe mapping has and attracting fixed point and, also, an attractive periodical point of period 2 and 4 respectively. (b) Values of fractal dimensions of Julia sets of the Potts-Bethe mapping for $z=11, Q=9$ and $\gamma=3$ near the first order phase transition point (a Yang-Lee zero) $\mu_{c}=0.74$.

\section{Properties of the Julia Sets of the Potts-Bethe Mapping}

In the following we will need some definitions from the theory of complex dynamical systems [19-22]. In a sense, the Julia set $J_{R}$ is a set of exceptional points for the iteration of $R: R^{n}(x)=R \circ \cdots \circ R(x) n$ times, $n=1,2, \ldots$. The complement of the Julia set is called Fatou set, $F_{R}=\bar{C} \backslash J_{R}$.

The Julia and Fatou sets of rational mapping $R$ have the following properties:

1. $J_{R}$ is not an empty set and contains more than countably many points.

2. Julia set is an invariant set, $R\left(J_{R}\right)=J_{R}=R^{-1}\left(J_{R}\right)$.

3. The set of all repelling periodic points of $R$ is dense in $J_{R}$.

4. If $J_{R}$ has interior points (i.e. there are points $a \in J_{R}$ such that for some $\left.\varepsilon>0\{x:|x-a|<\varepsilon\} \subset J_{R}\right)$ then $J_{R}=\bar{C}$.

5. If $R$ has only one attractive fixed point $a$ then $J_{R}=\partial A(a)$ and $A(a) \subset F_{R}$.

6. If $a$ and $b$ are attractive fixed points of $R$, then $(A(a) \cup A(b)) \subset F_{R}=\bar{C} \backslash J_{R}$ and $\partial A(a)=J_{R}=\partial A(b)$.

Here $\partial A(a)$ denotes the boundary of the basin of attraction $A(a)$ of attractive fixed point $a\left(\left|R^{\prime}(a)\right|<1\right.$ and $\left.R(a)=a\right)$. The basin of attraction $A(a)$ of attractive fixed point $a$ is defined as $A(a)=\left\{x \in \bar{C}: R^{k}(x) \rightarrow a\right.$ as $\left.k \rightarrow \infty\right\}$, i.e. $A(a)$ collects all points $x$ whose forward iterations approach $a$. For more details about Julia and Fatou sets we refer the reader to the literature on this subject [19-22]. 

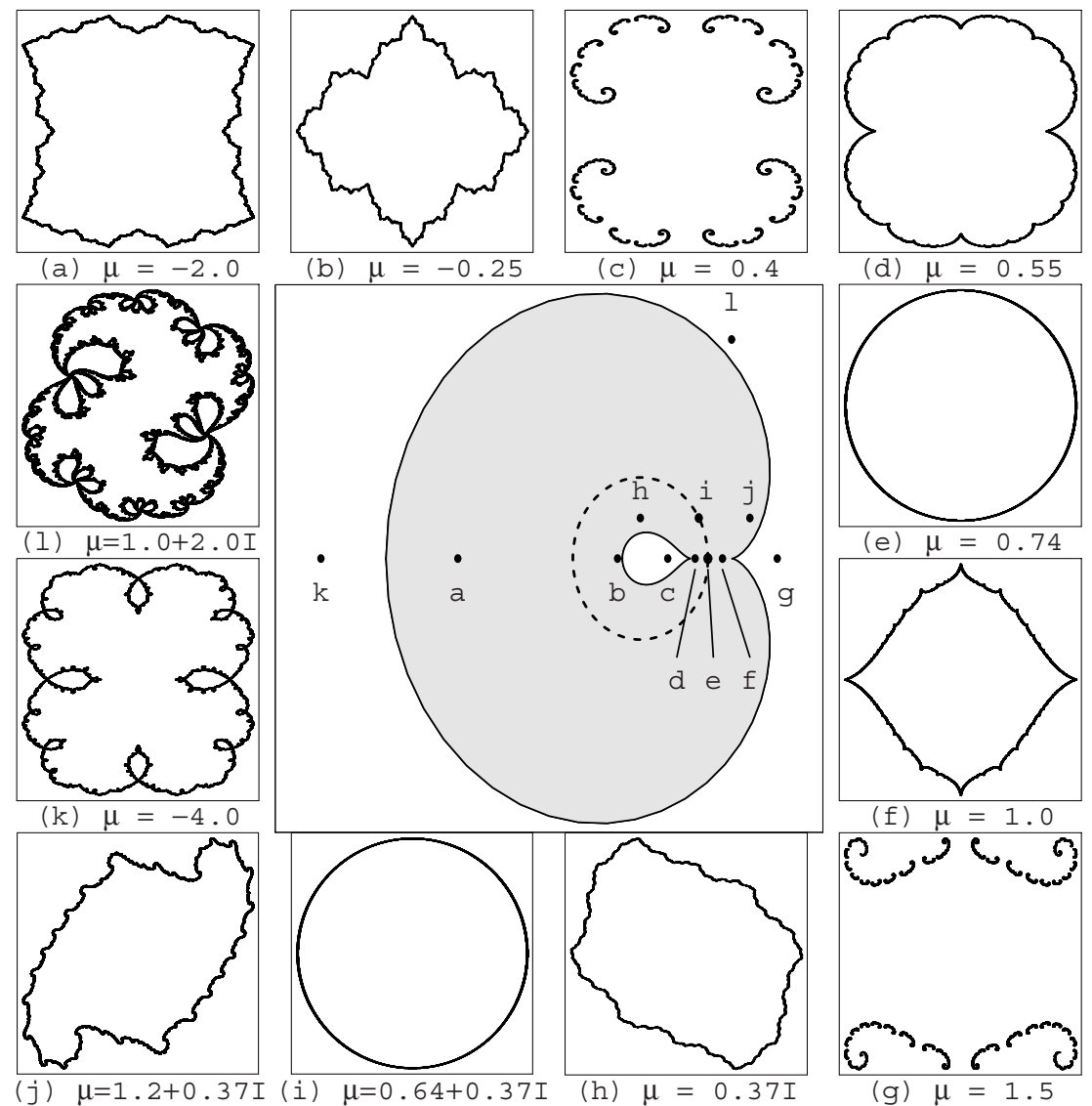

Fig. 4. The metastability region of the Potts-Bethe mapping for $z=11, Q=9$ and $\gamma=3$ is shown at the center of the figure. Pictures of Julia sets for values of magnetic field corresponding to points in the metastability region are plotted on figures (a)-(l). For an explanation of these pictures see the text.

Based on the properties of Julia and Fatou sets listed above we can study Julia sets of the Potts-Bethe mapping. In the Introduction we saw that for any temperature the metastability region decomposes the complex magnetic field plane into two components. Inside the metastability region the dynamics of the Potts-Bethe mapping $R$ is simple: $R$ has only two attracting fixed points. Outside the metastability region the dynamics of $R$ is quite complicated. In this case it is necessary to study the Mandelbrot-like set of the Potts-Bethe mapping. The algorithm for generating the Mandelbrot-like set is described in $[12,13]$. It based on testing the convergence of critical orbits of $R$ (See [20,21] for a definition of critical orbit). In Fig. 3.a we plotted the boundary of Mandelbrot-like set of Potts-Bethe mapping for $z=11, Q=9$ and $\gamma=3$. We have numerical evidence 
that there exists an attracting periodical point (a fixed point is a periodical point of period one) in white regions in Fig. 3.a. For example, at the points $k$ and $l$ inside the Mandelbrot-like set in Fig. 3.a the Potts-Bethe mapping has an attracting fixed point and an attracting periodical point of period 2 and 4 respectively. The corresponding Julia sets are closed Jordan curves and shown in Figs. 4.k and 1 respectively. At the points $c$ and $g$ outside the Mandelbrot-like set there is only one attractive fixed point and the corresponding Julia sets are Cantor sets Figs. 4.c and g respectively. From properties 4 and 5 we may conclude that for $\mu$ outside of the Mandelbrot-like set the Julia set is a Cantor set and its fractal dimension $D_{0}<2$. We used properties 2 and 3 to investigate Julia sets for this case. The numerical method we used is called the Modified Inverse Iteration Method [19]. The strategy of the method is: Starting from arbitrary initial point (it is better to use a repelling fixed point if it is known apriori) do inverse iterations of $R$. Usually the inverse function $R^{-1}$ is not unique and have several branches. Use a stochastic approach to determine the approximate location of the Julia set. Due to memory constrains it is easier to randomly choose between branches, than to use the full backward tree. This technique is well known [19]. After some iterations find the approximate box containing the Julia set. Use this box to put $J_{R}$ on a square lattice with small mesh size $\varepsilon$. Then, for any small box $B$ of the mesh, stop using points from $B$ for the inverse iteration, provided a certain number $N_{\max }$ of such points in B have been used. Optimal values of $\varepsilon$ and $N_{\max }$ depend on $R$. Therefore an interactive and adaptive algorithm is desirable.

Let us now consider the case when $\mu$ lies inside the metastability region. In this case $R$ has two attracting fixed points and our numerical calculations showed that the corresponding Julia sets are simply connected Jordan curves (Figs. 4.a, b, d-e, h-j). The numerical method we used based on property 6 of a Julia set and called Boundary Scanning Method. The goal is to follow the boundary of Julia set by pivoting through a chain of squares or triangles which approximate the boundary. We used triangles to approximate the boundary of Julia set because this algorithm was faster and gave the same results as with squares. Since in this case $R$ has two attractive fixed points so by checking the convergence of a given point $x$ on $x$-plane we can conclude to basin of attraction of which attractive fixed point it belongs. First one finds a triangle whose corner points belong to different basins of attraction. This means that this triangle intersects the Julia set. Then, one construct an adjacent triangle with the same property by reflecting the appropriate vertex across the center of the opposite side and so on, until one come to the starting triangle. This algorithm works perfectly if the Julia set is a closed curve.

For calculating the fractal dimension of a Julia set we used the box-counting method. This is a very useful method for many fractal structures. Let $N(\varepsilon)$ be the number of boxes of side $\varepsilon$ covering $J_{R}$, then

$$
D_{0}=-\lim _{\varepsilon \rightarrow 0} \frac{\log N(\varepsilon)}{\log \varepsilon} .
$$


In numerical calculations $\varepsilon$ is small but a finite number and $N(\varepsilon)$ has the form $N(\varepsilon)=G(\varepsilon) \varepsilon^{-D_{0}}$ where $G(\varepsilon)$ is a smooth and slowly varying function of $\varepsilon$. In order to discard the effect of $G(\varepsilon)$ usually for numerical calculations it is useful to use a little modified formula for calculating the fractal dimension

$$
D_{0}=-\lim _{\varepsilon \rightarrow 0, \varepsilon^{\prime} \rightarrow 0} \frac{\log \left(N(\varepsilon) / N\left(\varepsilon^{\prime}\right)\right)}{\log \left(\varepsilon / \varepsilon^{\prime}\right)} .
$$

The fractal dimensions of Julia sets of Potts-Bethe mapping are given in Fig. 3.b. From Fig. 3.b we see that the fractal dimension of Julia set for the value of magnetic field corresponding to a Yang-Lee zero (first order phase transition point) is a minimum as a function of magnetic field.

In the next section we give results of our numerical calculations.

\section{Results of calculations and concluding remarks}

Fractal dimensions of Julia sets of the Potts-Bethe mapping are studied numerically for complex values of $Q$. We used the Boundary Scanning Method (BSM) with triangulation of the plane for calculating the fractal dimensions of Julia set that is a closed Jordan curve and the Modified Inverse Iteration Method when the Julia set is a Cantor set. In this paper we give the program listing for the BSM algorithm. We run our program for several values of $Q$, temperature $z$, complex magnetic field $\mu$ and different values of $\varepsilon$ from formula (4). We found that Julia sets are circles centered at the origin with fractal dimension $D_{0}=1$ for values of magnetic field corresponding to the Yang-Lee zeros of the partition function(Figs. 4.e and i). For values of $\mu$ from the metastability region except the arc of Yang-Lee zeros the Julia set is a simply connected closed Jordan curve with fractal dimension $D_{0} \geq 1$ (Figs. 4 .a-d, f-h and $\mathrm{j}$ ). For values of $\mu$ outside the Mandelbrot-like set the Julia set is a Cantor set with fractal dimension $D_{0}<2$ (Figs. 4.c and g). The fractal dimension of Julia set for the value of magnetic field corresponding to Yang-Lee zeros is a minimum as a function of magnetic field (Fig. 3.b).

Below we give a brief description of the program. The function attractfp computes the attracting fixed point of the Bethe-Potts mapping (2) closest to the beginning of coordinates $(0,0)$. The method does not depend from the attractive fixed point we choose (See property 6 in Section 2.). For solving the polynomial equation we used zroots function from the book by Press et al. [23]. The function test computes whether the given point $x$ belongs to the basin of attraction of attractive fixed point attrfp or not. It returns 0 if the point belongs to a basin of attraction of attrfp and 1 if not. The remaining part of the main program computes the boundary of the Julia set and the number of triangles covering the Julia set for given $\varepsilon$. The $\mathrm{C}$ code of our program is given in Section 4 .

This work was supported by ANSEF Grant No. PS46. 


\section{Program Code}

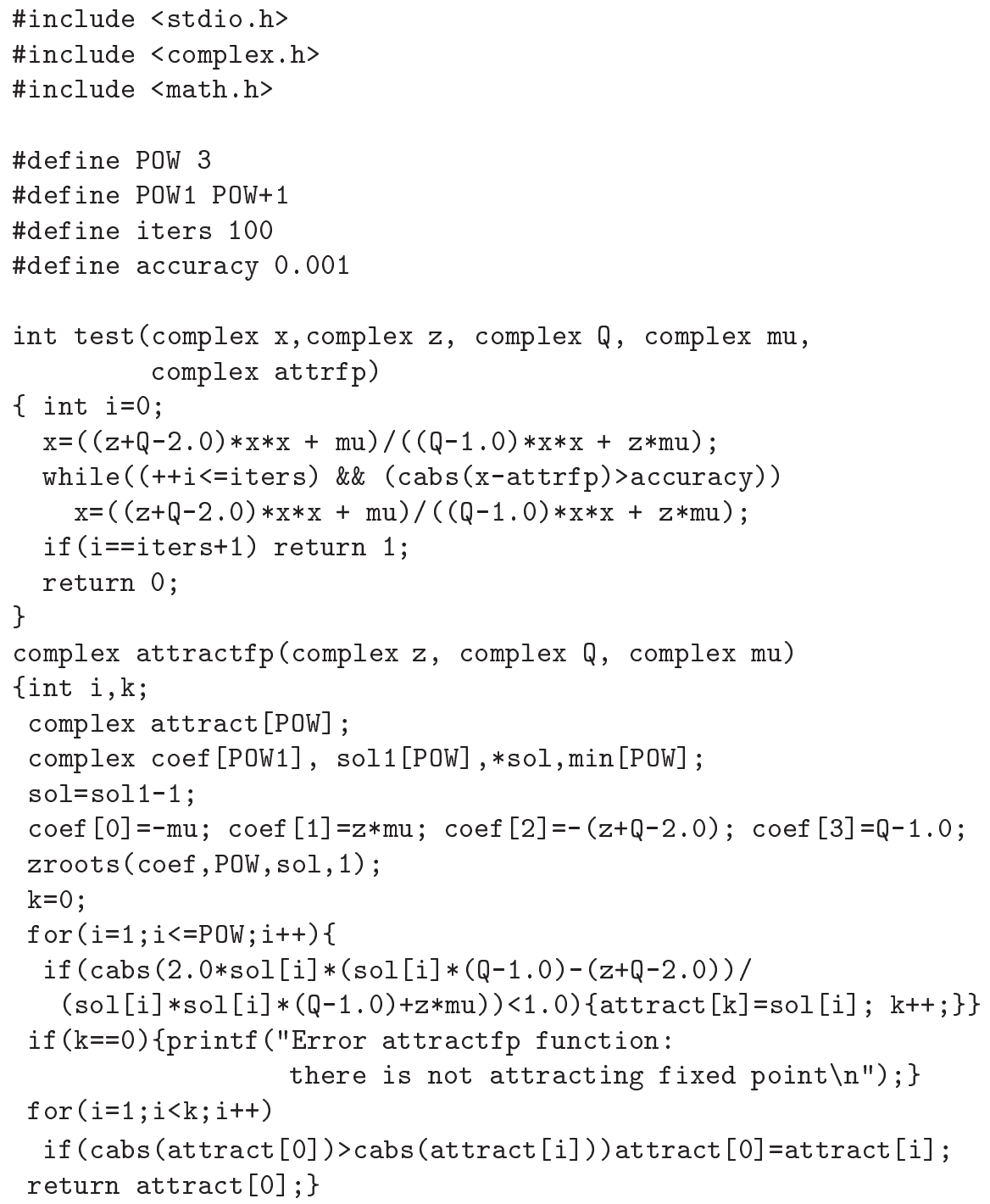




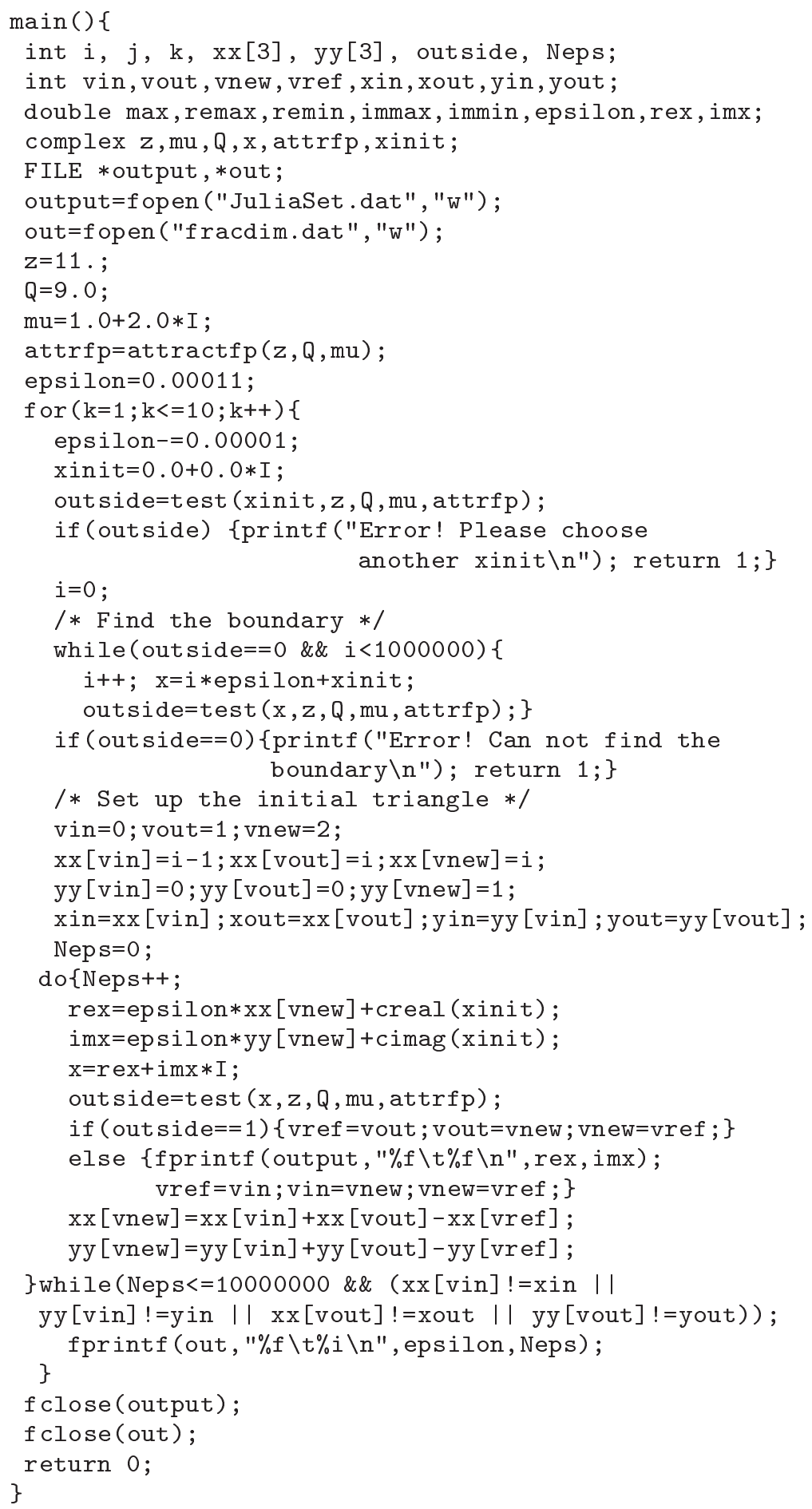




\section{References}

1. Wu F.Y.: The Potts model. Rev. Mod. Phys. 58 (1982) 235.

2. de Aguiar F.S. and Goulart-Rosa S. jr.: Phys. Lett. A 162 (1992) 232.

3. de Aguiar F. S., Bernardes L.B., and Goulart-Rosa S. Jr.: Journal of Stat. Phys. 64 (1991) 673.

4. Wagner F., Grensing D., and Heide J.: J. Phys. A: Math. Gen. 33 (2000) 929.

5. Ananikyan N.S. and Akheyan A.Z.: Zh. Eksp. Teor. Fiz. 107 (1995) 196.

6. Ghulghazaryan G.R., Ananikyan N.S., and Sloot P.M.A.: Phys. Rev. E 66 (2002) 046110 .

7. Peruggi F., di Liberto F., and Monroy G.: J. Phys. A: Math. Gen. 16 (1983) 811.

8. di Liberto F., Monroy G., and Peruggi F.: Z. Phys. B - Condensed Matter 66 (1987) 379.

9. Eggarter T.P.: Phys. Rev. B 9 (1974) 2989.

10. Müller-Hartmann E. and Zittartz J.: Phys. Rev. Lett. 33 (1974) 893.

11. Baxter R.J.: Exactly Solved Models in Statistical Mechanics. Academic Press, London (1981).

12. Ananikian N.S. and Ghulghazaryan R.G.: Phys. Lett. A 277, (2000) 249.

13. Ananikyan N. and Ghulghazaryan R.: J. Comput. Methods Sci. Eng. 2 (2002) 75.

14. Lee T.D. and Yang C.N.: Phys. Rev. 87: (1952) 404; 410.

15. Monroe J.: Phys. Lett. A 188 (1994) 80.

16. Biskup M., Borgs C., Chayes J.T., Kleinwaks L.J. and Kotecky R., Phys. Rev. Lett. 84, (2000) 4794.

17. Bosco F.A. and Goulart-Rosa S. jr.: Europhys Lett. 4 (1987) 1103; Comment by Monroe J.L.: Europhys. Lett. 29 (1995) 187.

18. Monroe J.L.: J. Phys. A: Math. Gen. 34 (2001) 6405.

19. Peitgen H.-O., Richter P.H.: The Beauty of Fractals. Springer-Verlag, Berlin Heidelberg (1986).

20. Blanchard P.: Bull. Am. Math. Soc. 11 (1984) 85.

21. Carleson L., Gamelin Th. W.: Complex Dynamics. Springer-Verlag, New York (1993).

22. Beardon A.: Iteration of Rational Functions. Springer-Verlag, New York (1991).

23. Press W.H., Teukolsky S.A., Vetterling W.T. and Flannery B.P.: Numerical Recipes in C. The Art of Scientific Computing. Cambridge University Press (1992). 